OPEN ACCESS

Edited by:

Zhihong Yang

Université de Fribourg, Switzerland

Reviewed by:

Xiu-Fen Ming

Université de Fribourg, Switzerland Carmelina Daniela Anfuso,

University of Catania, Italy

${ }^{*}$ Correspondence: Alejandro González-Candia alejandro.gonzalez@uoh.cl

Specialty section

This article was submitted to Integrative Physiology, a section of the journal

Frontiers in Physiology

Received: 31 May 2021

Accepted: 19 July 2021

Published: 19 August 2021

Citation:

Herrera EA and González-Candia A

(2021) Gestational Hypoxia and

Blood-Brain Barrier Permeability: Early

Origins of Cerebrovascular

Dysfunction Induced by Epigenetic Mechanisms.

Front. Physiol. 12:717550 doi: 10.3389/fphys.2021.717550

\section{Gestational Hypoxia and Blood-Brain Barrier Permeability: Early Origins of Cerebrovascular Dysfunction Induced by Epigenetic Mechanisms}

\author{
Emilio A. Herrera ${ }^{1}$ and Alejandro González-Candia ${ }^{2 *}$ \\ ${ }^{1}$ Laboratory of Vascular Function and Reactivity, Pathophysiology Program, ICBM, Faculty of Medicine, University of Chile, \\ Santiago, Chile, ${ }^{2}$ Institute of Health Sciences, University O'Higgins, Rancagua, Chile
}

Fetal chronic hypoxia leads to intrauterine growth restriction (IUGR), which is likely to reduce oxygen delivery to the brain and induce long-term neurological impairments. These indicate a modulatory role for oxygen in cerebrovascular development. During intrauterine hypoxia, the fetal circulation suffers marked adaptations in the fetal cardiac output to maintain oxygen and nutrient delivery to vital organs, known as the "brain-sparing phenotype." This is a well-characterized response; however, little is known about the postnatal course and outcomes of this fetal cerebrovascular adaptation. In addition, several neurodevelopmental disorders have their origins during gestation. Still, few studies have focused on how intrauterine fetal hypoxia modulates the normal brain development of the blood-brain barrier (BBB) in the IUGR neonate. The BBB is a cellular structure formed by the neurovascular unit (NVU) and is organized by a monolayer of endothelial and mural cells. The BBB regulates the entry of plasma cells and molecules from the systemic circulation to the brain. A highly selective permeability system achieves this through integral membrane proteins in brain endothelial cells. BBB breakdown and dysfunction in cerebrovascular diseases lead to leakage of blood components into the brain parenchyma, contributing to neurological deficits. The fetal brain circulation is particularly susceptible in IUGR and is proposed to be one of the main pathological processes deriving BBB disruption. In the last decade, several epigenetic mechanisms activated by IU hypoxia have been proposed to regulate the postnatal BBB permeability. However, few mechanistic studies about this topic are available, and little evidence shows controversy. Therefore, in this mini-review, we analyze the BBB permeability-associated epigenetic mechanisms in the brain exposed to chronic intrauterine hypoxia.

\footnotetext{
Keywords: chronic intrauterine hypoxia, brain endothelial dysfunction, cerebral circulation, fetal growth restriction, BBB permeability
}

\section{INTRODUCTION}

Fetal growth restriction (FGR) is a severe condition during pregnancy, where the fetus does not grow according to its potential as a result of an adverse uterine environment (Kingdom and Smith, 2000). Placental insufficiency is the predominant cause of FGR, leading to chronic fetal hypoxemia and intrauterine growth restriction (IUGR) (Kesavan and Devaskar, 2019). In addition, several 
babies are exposed to chronic hypoxia and IUGR due to pregnancy at a high altitude (Herrera et al., 2015). Intrauterine hypoxia induces an adaptive fetal redistribution of cardiac output, favoring vital organs such as the brain, known as the brain sparing effect (Giussani, 2016). In this scenario, the cerebral and heart circulations vasodilate, with a concomitant pronounced peripheral vasoconstriction (Villas-Bôas et al., 2008; Giussani, 2016). However, brain vasodilation does not ensure normal brain development in growth-restricted fetuses, and the neurodevelopmental outcomes will depend on the timing of hypoxia, the severity of IUGR, and the gestational age at delivery (Padilla et al., 2011; Baschat, 2014). Studies in animal models have demonstrated that gestational chronic hypoxia reduces the neuronal number and vascular and synaptic numbers in the hippocampus, impairing memory function in adult rats (Camm et al., 2021). In addition, IUGR may compromise cerebral vascular homeostasis by increased excitotoxicity, oxidative stress, and neuroinflammation (Miller et al., 2016; Sweeney et al., 2019). In structural terms, IUGR is associated with reduced brain and cortical volume, showing a reduced number of cells, and myelination shortages. These conditions are evidenced by less efficient networks with decreased long-range connections (Miller et al., 2016). Even more, some authors have proposed an association of fetal hypoxia to later neurodegenerative and neuropsychiatric disorders (Faa et al., 2014, 2016).

However, the principal factor of cerebrovascular diseases is the BBB breakdown, characterized by blood component infiltration, aberrant transport, and clearance of molecules into the central nervous system (CNS) (Yang and Rosenberg, 2011; Zhao et al., 2015). Structurally, the core of the BBB is a monolayer of brain endothelial cells; nevertheless, these cells cannot form a barrier on their own (Gastfriend et al., 2018). Indeed, the development of integrity characteristics in the cerebrovascular tree requires organized cells interactions from glial cells (i.e., astrocytes, microglia), pericytes, and neurons. Such a complex relationship implies the existence of a neurovascular unit (NVU) (Sweeney et al., 2019). The NVU represents a structural and functional multicellular interaction between cerebral parenchyma and brain circulation (Iadecola, 2017), establishing a highly selective BBB that favors cerebral homeostasis (Bell et al., 2020) (Figure 1). The complexity of this unit opens a wide and interesting field in the search for understanding the multiple processes that mediate cerebrovascular health.

\section{NEUROVASCULAR UNIT IN INTRAUTERINE GROWTH RESTRICTION}

The NVU plays various roles within the brain. This unit is responsible for the homeostasis and regulation of the cerebral blood flow in response to neuronal activity changes, known as neurovascular coupling (NVC) (Iadecola, 2017; Hendrikx et al., 2019). In addition, the same unit is in charge of protecting the CNS from harmful blood-borne and toxic substances (Blanchette and Daneman, 2015; Keaney and Campbell, 2015). From a structural view, three layers determine the barrier function in CNS, (i) the arachnoid barrier, (ii) the blood-cerebrospinal fluid barrier (BCSFB), and (iii) the blood-brain barrier (Tietz and Engelhardt, 2015). While the arachnoid barrier and the BCSFB have moderate permeability in the fetal, neonatal, and adult period, the $\mathrm{BBB}$ is the closest structure to the brain cells and hence, is considered the most important barrier (Benz and Liebner, 2020). The BBB is formed by endothelial cells that separate the capillary blood from the brain interstitial fluid and parenchyma, limiting transcellular and paracellular transport mechanisms through a differential expression of tight junctions (TJ), adherens junctions (AJ), and possibly gap junctions (GJ) in the inter-endothelial cleft (Figure 1). Also, the BBB comprises vascular smooth muscle cells, astrocytes, microglia, pericytes, and oligodendrocytes. These cells contribute to the permeability and integrity of the $\mathrm{BBB}$ through their intimate anatomical relationship (Liebner et al., 2018). However, the cross-talk between each cell type is partially understood, and our knowledge of neonatal BBB development remains incomplete.

Every constituent cell of the NVU contributes to the $\mathrm{BBBs}$ integrity, and any dysfunction might result in the barrier breakdown, with dramatic consequences such as neuroinflammation and neurodegeneration (Kempuraj et al., 2016; Sweeney et al., 2019). Although there is little or no evidence of the effects of IUGR on vascular permeability in human neonates exposed to hypoxia, there is plenty of data obtained from different animal models (Clancy et al., 2001, 2007; Kaur and Ling, 2008; Disdier and Stonestreet, 2020). Thus, the neonatal NVU increases the BBB's permeability by structural changes in the seal given by TJ, AJ, or GJ proteins. Brain endothelial cells contain low fenestration and selective rates of transcytosis mainly due to the high expression of the TJ proteins. TJ are a combination of transmembrane proteins (claudins and occludin) and cytoplasmic adapter proteins called zonula occludens (ZO) that interact with cytoskeleton filaments (Gonzalez-Candia et al., 2021). Decreases in TJ proteins expression have been reported after hypoxic exposition in neonatal brains. Specifically, hypoxia induces a decrease in claudin 5 and occludin protein levels, which increases the paracellular diffusion of solutes and ions across the BBB (Andersson et al., 2021). Furthermore, claudins decrease in the long term is associated with BBB breakdown and neurovascular disorders in humans (Tietz and Engelhardt, 2015). BBB functions have mainly focused on TJs; however, cadherin/catenin interaction, as AJ proteins, regulate cell-cell adhesion between endothelial cells, contributing to the overall junction arrangement and $\mathrm{BBB}$ integrity (Li et al., 2018). Vascular endothelial (VE)-cadherin is responsible for the assembly of AJ and is downregulated by BBB breakdown signaling events (Daneman and Prat, 2015). For instance, under neuroinflammatory conditions, $\mathrm{PI} 3 \mathrm{~K} \alpha$ triggers $\mathrm{TNF} \alpha$ signaling to cause VE-cadherin internalization, reducing the protein levels at junctions and impairing endothelial barrier function (Cain et al., 2010). Gestational or postnatal hypoxia can induce an unbalanced oxidative tone, as described elsewhere (Herrera et al., 2014; Villamor et al., 2019). The induction of the NADPH oxidase (NOX) system by proinflammatory mediators can generate $\mathrm{BBB}$ permeability by downregulation of proteins involved in intercellular junctions such as VE-cadherin, occludin, and claudin-5 (Rochfort et al., 2014). Another family 


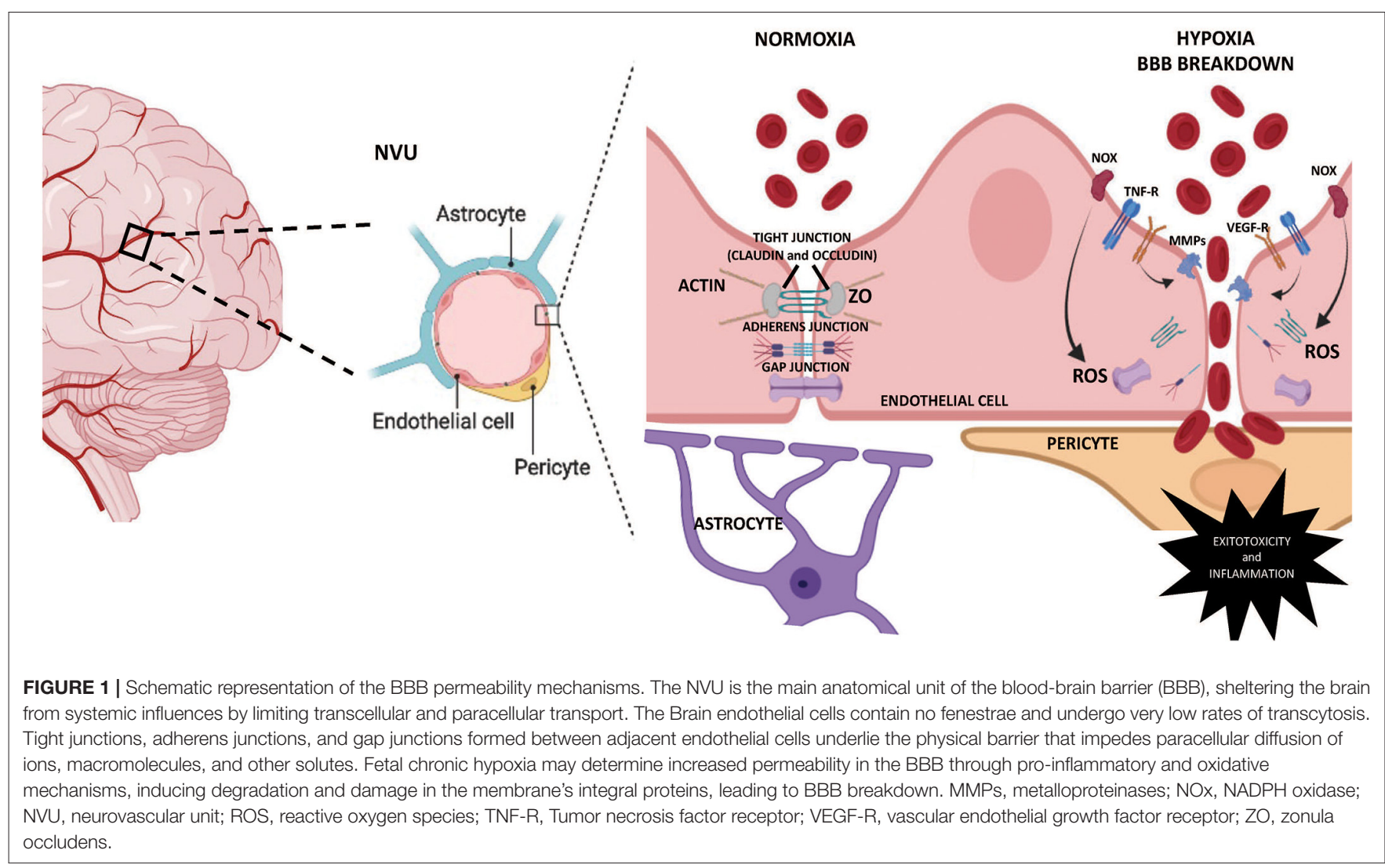

of proteins involved in the permeability of the $\mathrm{BBB}$ is the GJ, constituted by connexins. Connexin hemichannels have been implicated in the propagation of injury by hypoxia (Kim et al., 2017). Interestingly, neonatal hypoxia can negatively regulate the expression of connexin 43 (Davidson et al., 2013). In addition, the blockade of connexin 43 decreased oligodendrocyte death and recovered oligodendrocyte maturation in preterm fetuses exposed to perinatal asphyxia (Davidson et al., 2014).

Inflammatory mediators are critical for BBB disruption. Microglia, neurons, astrocytes, and endothelial cells can release proinflammatory cytokines and chemokines, modulating adhesion molecules and transmigration of activated immune cells into the brain parenchyma (Jickling et al., 2015; Huang et al., 2016). In endothelial cells, proinflammatory molecules regulate the expression of adhesion molecules such as intercellular adhesion molecule 1 (ICAM-1) and vascular cell adhesion protein 1 (VCAM-1), physiologically expressed at low levels in the $\mathrm{BBB}$. However, their expression is increased in response to hypoxia, increasing the extravasation of molecules into the brain parenchyma (Kong et al., 2018).

In addition, the increase in cellular levels of TNF- $\alpha$ and IL-1 $\beta$ has been related to the decrease in occludin expression and ZO-1 and 2 in the hypoxic brain (Rochfort and Cummins, 2015; Abdullah et al., 2018). This causes an increased paracellular permeability, modulation of transcytosis, and endocytotic transport mechanisms, leading to changes in transcellular transport and inflammatory damage in the brain parenchyma (Sweeney et al., 2019). Besides, reactive glial cells, members of the NVU, are likely to contribute to the permeability of the BBB observed in cerebral hypoxia through downregulation of paracellular proteins such as Claudin-5 (CLDN5), occludin, and ZO-1 (Obermeier et al., 2013). On the other hand, hypoxiainduced vascular endothelial growth factor (VEGF) type 2 receptor (VEGFR-2) pathway activation, increasing permeability in the brain microvascular endothelium by decreasing junctional proteins claudin-5, occludin, and ZO-1 (Castañeda-Cabral et al., 2020). Besides, in postnatal cerebral ischemia, VEGF may affect $\mathrm{BBB}$ damage by inducing metalloproteinases (MMP)-2 expression, increasing the BBB permeability by brain endothelial dysfunction (Shen et al., 2018).

Oxidative stress has a critical role in BBB breakdown in different neurological conditions (Olmec and Ozyurt, 2012). Although the hypoxia generated in IUGR is sufficient to generate a redox imbalance (Myatt and Cui, 2004; Herrera et al., 2014), direct evidence in human or animal models of BBB permeability is associated with IUGR remains to be elucidated. CNS contains several sources of ROS, such as NOX, uncoupling of the mitochondrial electron transport chain, xanthine oxidase isoform, and uncoupled nitric oxide synthase (NOS) (Warner et al., 2004). The NOX family seems to be a principal source of oxidative stress in the hypoxic brain through the generation of superoxide (O2•-) radicals (Yang et al., 2019). The predominant isoform is the NOX2 in brain endothelial cells, and it has been observed that the Nox2- knockout mice induce less MMP9 and diminished expression of occludin, a critical protein of the BBB permeability (Liu et al., 2011). In addition, ROS 
generated by NOX can act as activators of MMPs (Li et al., 2018), thus enhancing their proteolytic degradation to the BBB. Among MMP family members, MMP- 2 and 9 possess a substrate specificity for fibronectin, laminin, collagen fibers, and TJ, all of them structural components of the BBB. Interestingly, these proteins can be induced by hypoxia (Rosenberg and Yang, 2007).

\section{PERINATAL PROGRAMMING OF THE NVU: POTENTIAL EPIGENETIC MECHANISMS}

Adverse environmental conditions during development, such as prenatal hypoxia, can increase the risk of diseases in adulthood, such as vascular and parenchymal brain diseases (Berson et al., 2018). Basic and translational studies have demonstrated that epigenetic programming of gene patterns in response to gestational stress have a critical function in the fetal origins of neurological cells dysfunction (Ducsay et al., 2018). In particular, during gestational hypoxia, the epigenetic programming of genes determines the functional outcome of the genome (Ducsay et al., 2018). Epigenetics as heritable patterns in gene expression which are not associated with DNA sequence alteration (Smith et al., 2016). The epigenetic mechanisms include methylation and/or demethylation of DNA, post-translational modifications of histones, and non-coding RNAs such as microRNAs (Casanello et al., 2016; Ducsay et al., 2018; Zeng and Chen, 2018). Epigenetic events respond to endogenous and exogenous signals, having central roles in regulating appropriate sets of gene expression (Zeng and Chen, 2018). Epigenetic modifications serve as remembrance in early life stages, that can induce long-term changes in gene expression, which may induce disease in later postnatal life (Ducsay et al., 2018).

Hypoxic stress activates multiple epigenetic mechanisms in the fetal brain that increase the vulnerability for neurodevelopment disturbances in adult offspring (Ma et al., 2014; Faa et al., 2016), such as increased vulnerability to ischemic or hypoxic insults (Li et al., 2012; Gonzalez-Rodriguez et al., 2014), disruption of the normal endocrine axis (Wood et al., 2014), and increased risks for adult cardiovascular disease (Ducsay et al., 2018). The mechanisms underlying the effects of chronic fetal hypoxia and IUGR on epigenetic programming of the fetal brain endothelial cells or NVU has not been studied. However, the effects induced by hypoxia and oxidative stress in the fetal brain suggest the involvement of epigenetic mechanisms (Camm et al., 2021).

DNA methylation regulates the accessibility of DNA to the transcription machinery modifying the chromatin state. This DNA methylation is generated by a group of enzymes known as DNA methyltransferases (DNMTs) (Ducsay et al., 2018); composed by three principal isoforms: maintenance DNMTs (DNMT1) and de novo DNMTs (DNMT3a and DNMT3b) (Moore et al., 2013). However, no mechanisms have been proposed to demonstrate the DNA methylation events during gestational hypoxia, that may regulate the expression of $\mathrm{BBB}$ structural proteins and permeability. In this sense, the effects of hypoxia and IUGR can only be extrapolated in neonatal studies or adult models of cerebral ischemia. In models of cerebral hypoxia-ischemia, an increase of DNA methylation was described as an increase in global DNA methylation in the murine cerebral hemispheres, in the promoter of tissue inhibitors of MMP-2 (TIMP2). Increased MMP-2 and MMP-9 expression and activity can affect $\mathrm{BBB}$ permeability by proteolysis of extracellular matrix and structural proteins in brain endothelial cells, increasing the BBB breakdown (Figure 2) (Yang et al., 2007; Wang et al., 2012). Late gestational maternal hypoxia in rats induce hypomethylation in the fetal brain by a mechanism dependent on HIF-1 $\alpha$ expression (Li et al., 2016). This is relevant as the HIF-related pathway is recognized as the primary sensor and effector for hypoxic cellular adaptation in the fetus (Herrera et al., 2014). Hypomethylation induced by maternal hypoxia increased the vulnerability to subsequent postnatal hypoxia and worsened neurobehavioral outcomes in rat pups (Chen et al., 2008; Li et al., 2016). Interestingly, some authors have shown that HIF-1 expression levels and its transcriptional activity are under strong epigenetic regulation (Nguyen et al., 2013; Ma et al., 2014) and others that HIF-1 itself controls the expression of several epigenetic regulators (Bustelo et al., 2020). The role of HIF in BBB functional programming during fetal hypoxia is still unknown and needs further study.

Histone modification by acetylation and deacetylation plays a central role in chromatin remodeling and epigenetic regulation. In particular, histone deacetylases (HDAC) are potential therapeutic targets in different neurological conditions (Gräff and Tsai, 2013). In a recent study, the treatment with a HDAC inhibitor in mice subjected to cerebral ischemia leads to an enhanced expression of the TJ proteins ZO-1, Occludin, and Claudin-5 in brain endothelial cells, further decreasing the BBB permeability (Su et al., 2020). Conversely, hypoxia and glucose deprivation in the brain promotes HDAC9 expression in endothelial cells, which has been associated to decreased expression of ZO-1, claudin-5, and occludin (Shi et al., 2016). These findings demonstrate the effect of hypoxia on the post-translational modifications of histones in the regulation of proteins involved in the maintenance of the BBB structure and that these mechanisms may be determining the dysfunction of the BBB induced by the hypoxia.

Another mechanism of epigenetic regulation is mediated by microRNAs, which cause the degradation of genes involved in the development and progression of BBB dysfunction (Figure 2) (Ma et al., 2020). Currently, there are no data relating to IUGR and microRNA regulating $\mathrm{BBB}$ structure and function; however, evidence in adult pathophysiology may give some clues about microRNAs and BBB disruption. Hypoxic-ischemic models in adult animals have shown that microRNAs can directly or indirectly degrade BBB proteins. In this sense, it has been reported that miR-132 is negatively regulated by hypoxia, which increases MMP-9 activity, which degrades TJ proteins in brain endothelial cells or extracellular matrix components in the NVU, favoring an increased permeability of the BBB (Cichon et al., 2014). There are significant correlations between microRNAs and TJs by hypoxia in adult models (Toyama et al., 2017). For instance, miR-125-5p has a critical role in 


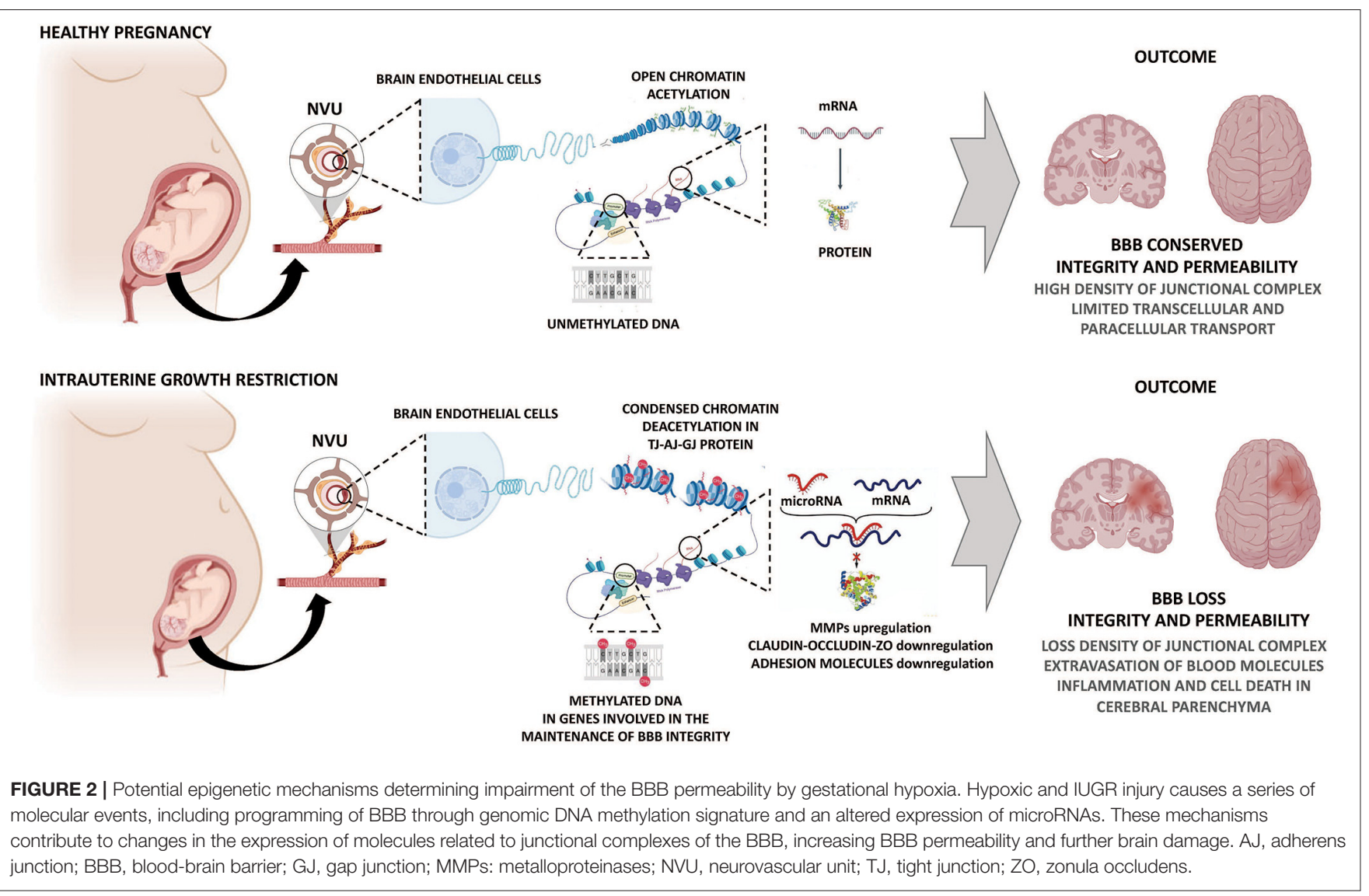

the brain endothelial tightness during an inflammatory response (Toyama et al., 2017). Part of this response involves specific mRNA targets of miR-125-5p by down-regulating Claudin1 and Claudin-5, and disrupting adhesion molecules in BBB (Toyama et al., 2017). Furthermore, cerebral endothelial miR144 downregulates claudin-5, Claudin-12, occludin, and ZO-1, $\mathrm{ZO}-2$, and $\mathrm{ZO}-3$ in a model of $\mathrm{BBB}$ permeability associated with a blood-tumor barrier (Cai et al., 2017). Cerebral ischemia triggers an enhanced expression of miR126, which is considered endothelial-specific. miR126 is one of the most studied microRNAs that regulates vascular inflammation. miR126 downregulates the expression of the ICAM-1 and VCAM1 molecules and controls inflammatory cells extravasation into the brain in BBB dysfunction models (Stamatovic et al., 2016). These data suggest that epigenetic mechanisms define and regulate the vascular responses to pathological stimuli such as chronic hypoxia (Figure 2). However, evidence from fetal exposure to hypoxia leading to epigenetic modifications remains elusive.

\section{CONCLUSION}

Chronic deprivation of oxygen during gestation dramatically impacts fetal brain development. Gestational hypoxia can act through an altered epigenetic fashion to compromise placental and vascular function (Gheorghe et al., 2010; Herrera et al., 2014; Fajersztajn and Veras, 2017; Soares et al., 2017). However, advances in understanding how gestational hypoxia induces variations in the expression of proteins involved in the integrity of the cerebrovascular network remain widely unexplored. BBB permeability is a major factor determining the cause, progression, outcome, and therapeutic effectiveness of different neurological impairments in postnatal life. Therefore, fetal programming of $\mathrm{BBB}$ permeability by hypoxia and IUGR pose a unique challenge to the scientific community in searching for involved mechanisms and effective clinical treatment to prevent detrimental postnatal outcomes.

\section{AUTHOR CONTRIBUTIONS}

AG-C and EH drafted and edited the manuscript. Both authors contributed to the article and approved the submitted version.

\section{FUNDING}

This work was funded by Fondecyt de Inicio grant No 11200798 and Fondecyt Regular grant No 1201283. 


\section{REFERENCES}

Abdullah, W., Tripathi, D., and Ronaldson, P. T. (2018). Blood-brain barrier dysfunction in ischemic stroke: targeting tight junctions and transporters for vascular protection. Am. J. Physiol. Cell Physiol. 315, C343-C356. doi: 10.1152/ajpcell.00095.2018

Andersson, E. A., Mallard, C., and Ek, C. J. (2021). Circulating tight-junction proteins are potential biomarkers for blood-brain barrier function in a model of neonatal hypoxic/ischemic brain injury. Fluids Barriers CNS 18:7. doi: 10.1186/s12987-021-00240-9

Baschat, A. A. (2014). Neurodevelopment after fetal growth restriction. Fetal Diagn Ther. 36, 136-142. doi: 10.1159/000353631

Bell, A. H., Miller, S. L., Castillo-Melendez, M., and Malhotra, A. (2020). The neurovascular unit: effects of brain insults during the perinatal period. Front. Neurosci. 13:1452. doi: 10.3389/fnins.2019.01452

Benz, F., and Liebner S. (2020). "Structure and function of the blood-brain barrier (bbb)," in Handbook of Experimental Pharmacology (Berlin; Heidelberg: Springer). doi: 10.1007/164_2020_404

Berson, A., Nativio, R., Berger, S. L., and Bonini, N. M. (2018). Epigenetic regulation in neurodegenerative diseases. Trends Neurosci. 41, 587-598. doi: 10.1016/j.tins.2018, 05.005

Blanchette, M., and Daneman, R. (2015). Formation and maintenance of the BBB. Mech. Dev. 138, 8-16. doi: 10.1016/j.mod.2015. 07.007

Bustelo, M., Barkhuizen, M., van den Hove, D. L. A., Steinbusch, H. W. M., Bruno, M. A., Loidl, C. F., et al. (2020). Clinical implications of epigenetic dysregulation in perinatal hypoxicischemic brain damage. Front. Neurol. 11:483. doi: 10.3389/fneur.2020. 00483

Cai, W., Zhang, K., Li, P., Zhu, L., Xu, J., Yang, B., et al. (2017). Dysfunction of the neurovascular unit in ischemic stroke and neurodegenerative diseases: an aging effect. Ageing Res. Rev. 34:77-87. doi: 10.1016/j.arr.2016. 09.006

Cain, R. J., Vanhaesebroeck, B., and Ridley, A. J. (2010). The PI3K p110alpha isoform regulates endothelial adherens junctions via Pyk2 and Rac1. J. Cell Biol. 188, 863-876. doi: 10.1083/jcb.2009 07135

Camm, E. J., Cross, C. M., Kane, A. D., Tarry-Adkins, J. L., Ozanne, S. E., and Giussani, D. A. (2021). Maternal antioxidant treatment protects adult offspring against memory loss and hippocampal atrophy in a rodent model of developmental hypoxia. FASEB J. 35:e21477. doi: 10.1096/fj.20200 2557RR

Casanello, P., Herrera, E. A., and Krause, B. J. (2016). Epigenetic programming of cardiovascular disease by perinatal hypoxia and fetal growth restriction. Hypoxia Human Dis. 17, 330-347. doi: 10.5772/66740

Castañeda-Cabral, J. L., Colunga-Durán, A., Ureña-Guerrero, M. E., BeasZárate, C., Nuñez-Lumbreras, M. L. A., Orozco-Suárez, S., et al. (2020). Expression of VEGF- and tight junction-related proteins in the neocortical microvasculature of patients with drug-resistant temporal lobe epilepsy. Microvasc. Res. 132:104059. doi: 10.1016/j.mvr.2020. 104059

Chen, W., Jadhav, V., Tang, J., and Zhang, J. H. (2008). HIF-1 alpha inhibition ameliorates neonatal brain damage after hypoxic-ischemic injury. Acta Neurochir. Suppl. 102, 395-399. doi: 10.1007/978-3-21185578-2_77

Cichon, C., Sabharwal, H., Rüter, C., and Schmidt, M. A. (2014). MicroRNAs regulate tight junction proteins and modulate epithelial/endothelial barrier functions. Tissue Barriers 2:e944446. doi: 10.4161/21688362.2014. 944446

Clancy, B., Darlington, R. B., and Finlay, B. L. (2001). Translating developmental time across mammalian species. Neuroscience $\quad 105, \quad 7-17 . \quad$ doi: $\quad 10.1016 / \mathrm{S} 0306-4522(01)$ 00171-3

Clancy, B., Finlay, B. L., Darlington, R. B., and Arland, K. J. S. (2007). Extrapolating brain development from experimental species to humans. Neurotoxicology 28 , 931-937. doi: 10.1016/j.neuro.2007.01.014
Daneman, R., and Prat, A. (2015). The blood-brain barrier. Cold Spring Harb. Perspect. Biol. 7:a020412. doi: 10.1101/cshperspect.a020412

Davidson, J. O., Drury, P. P., Green, C. R., Nicholson, L. F., Bennet, L., and Gunn, A. J. (2014). Connexin hemichannel blockade is neuroprotective after asphyxia in preterm fetal sheep. PLoS ONE 9:e96558. doi: 10.1371/journal.pone.0096558

Davidson, J. O., Green, C. R., Nicholson, L. F., Bennet, L., and Gunn, A. J. (2013). Connexin hemichannel blockade is neuroprotective after, but not during, global cerebral ischemia in near-term fetal sheep. Exp. Neurol. 248, 301-308. doi: 10.1016/j.expneurol.2013.06.026

Disdier, C., and Stonestreet, B. S. (2020). Hypoxic-ischemicrelated cerebrovascular changes and potential therapeutic strategies in the neonatal brain. J Neurosci Res. 98, 1468-1484. doi: 10.1002/jnr.24590

Ducsay, C. A., Goyal, R., Pearce, W. J., Wilson, S., Hu, X. Q., and Zhang, L. (2018). Gestational hypoxia and developmental plasticity. Physiol. Rev. 98, 1241-1334. doi: 10.1152/physrev.00043.2017

Faa, G., Manchia, M., Pintus, R., Gerosa, C., Marcialis, M. A., and Fanos, V. (2016). Fetal programming of neuropsychiatric disorders. Birth Defects Res. C Embryo Today. 108, 207-223. doi: 10.1002/bdrc.21139

Faa, G., Marcialis, M. A., Ravarino, A., Piras, M., Pintus, M. C., and Fanos, V. (2014). Fetal programming of the human brain: is there a link with insurgence of neurodegenerative disorders in adulthood?. Curr. Med. Chem. 21, 3854-3876. doi: 10.2174/0929867321666140601163658

Fajersztajn, L., and Veras, M. M. (2017). Hypoxia: from placental development to fetal programming. Birth Defects Res. 109, 1377-1385. doi: 10.1002/bdr2.1142

Gastfriend, B. D., Palecek, S. P., and Shusta, E. V. (2018). Modelling the bloodbrain barrier: beyond the endothelial cells. Curr. Opin. Biomed. Eng. 5, 6-12. doi: 10.1016/j.cobme.2017.11.002

Gheorghe, C. P., Goyal, R., Mittal, A., and Longo, L. D. (2010). Gene expression in the placenta: maternal stress and epigenetic responses. Int. J. Dev. Biol. 54, 507-523. doi: $10.1387 / \mathrm{ijdb} .082770 \mathrm{cg}$

Giussani, D. A. (2016). The fetal brain sparing response to hypoxia: physiological mechanisms. J. Physiol. 594, 1215-1230. doi: 10.1113/JP271099

Gonzalez-Candia, A., Rogers, N. K., and Castillo, R. L. (2021). "Blood-brain barrier dysfunction in the detrimental brain function," in Connectivity and Functional Specialization in the Brain, ed T. Heinbockel (London: Intechope). doi: 10.5772/intechopen.94572

Gonzalez-Rodriguez, P. J., Xiong, F., Li, Y., Zhou, J., and Zhang, L. (2014). Fetal hypoxia increases vulnerability of hypoxicischemic brain injury in neonatal rats: role of glucocorticoid receptors. Neurobiol. Dis. 65, 172-179. doi: 10.1016/j.nbd.2014. 01.020

Gräff, J., and Tsai, L. H. (2013). The potential of HDAC inhibitors as cognitive enhancers. Аnnu. Rev. Pharmacol. Toxicol. 53, 311-330. doi: 10.1146/annurev-pharmtox-011112-140216

Hendrikx, D., Smits, A., Lavanga, M., De Wel, O., Thewissen, L., Jansen, K., et al. (2019). Measurement of neurovascular coupling in neonates. Front. Physiol. 10:65. doi: 10.3389/fphys.2019.00065

Herrera, E.A., Krause, B., Ebensperger, G., Reyes, R.V., Casanello, P., ParraCordero, M., et al. (2014). The placental pursuit for an adequate oxidant balance between the mother and the fetus. Front. Pharmacol. 24:149. doi: 10.3389/fphar.2014.00149

Herrera, E. A., Farías, J. G., Ebensperger, G., Reyes, R. V., Llanos, A. J., and Castillo, R. L. (2015). Pharmacological approaches in either intermittent or permanent hypoxia: a tale of two exposures. Pharmacol Res. 101, 94-101. doi: 10.1016/j.phrs.2015. 07.011

Huang, T., Gao, D., Hei, Y., Zhang, X., Chen, X., and Fei, Z. (2016). D-allose protects the blood brain barrier through PPAR $\gamma$-mediated anti-inflammatory pathway in the mice model of ischemia reperfusion injury. Brain Res. 1642, 478-486. doi: 10.1016/j.brainres.2016. 04.038

Iadecola, C. (2017). The neurovascular unit coming of age:a journey through neurovascular coupling in health and disease. Neuron 96, 17-42. doi: 10.1016/j.neuron.2017.07.030

Jickling, G. C., Liu, D., Ander, B. P., Stamova, B., Zhan, X., and Sharp, F. R. (2015). Targeting neutrophils in ischemic stroke: translational insights from experimental studies. $J$. 
Cereb. Blood Flow Metab. 35, 888-901. doi: 10.1038/jcbfm. 2015.45

Kaur, C., and Ling, E. A. (2008). Blood brain barrier in hypoxic-ischemic conditions. Curr. Neurovas. Res. 5, 71-81. doi: 10.2174/156720208783565645

Keaney, J., and Campbell, M. (2015). The dynamic bloodbrain barrier. FEBS J. 282, 4067-4079. doi: 10.1111/febs. 13412

Kempuraj, D., Thangavel, R., Natteru, P. A., Selvakumar, G. P., Saeed, D., Zahoor, H., et al. (2016). Neuroinflammation induces neurodegeneration. J. Neurol. Neurosurg. Spine 1:1003.

Kesavan, K., and Devaskar, S. U. (2019). Intrauterine growth restriction: postnatal monitoring and outcomes. Pediatr. Clin. North Am. 66, 403-423. doi: 10.1016/j.pcl.2018. 12.009

Kim, Y., Davidson, J. O., Green, C. R., Nicholson, L. F. B., O'Carroll, S. J., and Zhang, J. (2017). Connexins and Pannexins in cerebral ischemia. Biochim. Biophys. Acta Biomembr. 1860, 224-236. doi: 10.1016/j.bbamem.2017.03.018

Kingdom, J., and Smith, G. (2000). "Diagnosis and management of IUGR," in Intrauterine Growth Restriction A Etiology and Management, eds J. Kingdom and P. Baker (London: v), 257-273. doi: 10.1007/978-1-4471-0735-4_13

Kong, D. H., Kim, H., Kim, M. R., Jang, J. H., and Lee, S. (2018). Emerging roles of Vascular Cell Adhesion Molecule-1 (VCAM-1) in immunological disorders and cancer. Int. J. Mol. Sci. 19:1057. doi: 10.3390/ijms19041057

Li, W., Chen, Z., Chin, I., Chen, Z., and Dai, H. (2018). The role of VE-cadherin in blood-brain barrier integrity under central nervous system pathological conditions. Curr. Neuropharmacol. 16, 1375-1384. doi: 10.2174/1570159X166661802 22164809

Li, Y., Gonzalez, P., and Zhang, L. (2012). Fetal stress and programming of hypoxic/ischemic-sensitive phenotype in the neonatal brain: mechanisms and possible interventions. Prog Neurobiol. 98, 145-165. doi: 10.1016/j.pneurobio.2012.05.010

Li, Y., Ma, Q., Halavi, S., Concepcion, K., Hartman, R. E., Obenaus, A., et al. (2016). Fetal stress-mediated hypomethylation increases the brain susceptibility to hypoxic-ischemic injury in neonatal rats. Exp. Neurol. 275, 1-10. doi: 10.1016/j.expneurol.2015.10.007

Liebner, S., Dijkhuizen, R. M., Reiss, Y., Plate, K. H., Agalliu, D., and Constantin, G. (2018). Functional morphology of the blood-brain barrier in health and disease. Acta Neuropathol. 135: 311-336. doi: 10.1007/s00401-0181815-1

Liu, W., Chen, Q., Liu, J., and Liu, K. J. (2011). Normobaric hyperoxia protects the blood brain barrier through inhibiting Nox2 containing NADPH oxidase in ischemic stroke. Med. Gas Res. 1:22. doi: 10.1186/2045-9912-1-22

Ma, F., Zhang, X., and Yin, K. J. (2020). MicroRNAs in central nervous system diseases: a prospective role in regulating blood-brain barrier integrity. Exp. Neurol. 323:113094. doi: 10.1016/j.expneurol.2019. 113094

Ma, Q., Xiong, F., and Zhang, L. (2014). Gestational hypoxia and epigenetic programming of brain development disorders. Drug Discov. Today 19, 1883-1896. doi: 10.1016/j.drudis.2014.09.010

Miller, S. L., Huppi, P. S., and Mallard, C. (2016). The consequences of fetal growth restriction on brain structure and neurodevelopmental outcome. J. Physiol. 594, 807-823. doi: 10.1113/JP271402

Moore, L. D., Le, T., and Fan, G. (2013). DNA methylation and its basic function. Neuropsychopharmacology 38, 23-38. doi: 10.1038/npp.2012.112

Myatt, L., and Cui, X. (2004). Oxidative stress in the placenta. Histochem. Cell Biol. 122, 369-382. doi: 10.1007/s00418-004-0677-x

Nguyen, M. P., Lee, S., and Lee, Y. M. (2013). Epigenetic regulation of hypoxia inducible factor in diseases and therapeutics. Arch. Pharm. Res. 36, 252-263. doi: 10.1007/s12272-013-0058-x

Obermeier, B., Daneman, R., and Ransohoff, R. M. (2013). Development, maintenance and disruption of the blood-brain barrier. Nat. Med. 19, 1584-1596. doi: 10.1038/nm.3407

Olmec, I., and Ozyurt, H. (2012). Reactive oxygen species and ischemic cerebrovascular disease. Neurochem. Int. 60, 208-212. doi: 10.1016/j.neuint.2011.11.009
Padilla, N., Falcon, C., Sanz-Cortes, M., Figueras, F., Bargallo, N., Crispi, F., et al. (2011). Differential effects of intrauterine growth restriction on brain structure and development in preterm infants: amagnetic resonance imaging study. Brain Res. 1382, 98-108. doi: 10.1016/j.brainres.2011.01.032

Rochfort, K. D., Collins, L. E., Murphy, R. P., and Cummins, P. M. (2014). Downregulation of blood-brain barrier phenotype by proinflammatory cytokines involves NADPH oxidase-dependent ROS generation: consequences for interendothelial adherens and tight junctions. PLoS ONE 9:e101815. doi: 10.1371/journal.pone. 0101815

Rochfort, K. D., and Cummins, P. M. (2015). Cytokine-mediated dysregulation of zonula occludens-1 properties in human brain microvascular endothelium. Microvasc Res. 100, 48-53. doi: 10.1016/j.mvr.2015. 04.010

Rosenberg, G. A., and Yang, Y. (2007). Vasogenic edema due to tight junction disruption by matrix metalloproteinases in cerebral ischemia. Neurosurg. Focus 22:E4. doi: 10.3171/foc.2007.22.5.5

Shen, Y., Gu, J., Liu, Z., Xu, C., Qian, S., Zhang, X., et al. (2018). Inhibition of HIF$1 \alpha$ reduced blood brain barrier damage by regulating MMP- 2 and VEGF during acute cerebral ischemia. Front. Cell. Neurosci. 12:288. doi: 10.3389/fncel.2018. 00288

Shi, W., Wei, X., Wang, Z., Han, H., Fu, Y., Liu, J., et al. (2016). HDAC9 exacerbates endothelial injury in cerebral ischaemia/reperfusion injury. J. Cell. Mol. Med. 20, 1139-1149. doi: $10.1111 / \mathrm{jcmm} .12803$

Smith, Z. D., Sindhu, C., and Meissner, A. (2016). Molecular features of cellular reprogramming and development. Nat. Rev. Mol. Cell Biol. 17, 139-154. doi: $10.1038 / \mathrm{nrm} .2016 .6$

Soares, M. J., Iqbal, K., and Kozai, K. (2017). Hypoxia and placental development. Birth Defects Res. 109, 1309-1329. doi: 10.1002/bdr2.1135

Stamatovic, S. M., Johnson, A. M., Keep, R. F., and Andjelkovic, A. V. (2016). Junctional proteins of the blood-brain barrier: new insights into function and dysfunction. Tissue Barriers 4:e1154641. doi: 10.1080/21688370.2016. 1154641

Su, L., Liang, D., Kuang, S. Y., Dong, Q., Han, X., and Wang, Z. (2020). Neuroprotective mechanism of TMP269, a selective class IIA histone deacetylase inhibitor, after cerebral ischemia/reperfusion injury. Neural. Regen. Res. 15, 277-284. doi: 10.4103/1673-5374. 265562

Sweeney, M. D., Zhao, Z., Montagne, A., Nelson, A. R., and Zlokovic, B. V. (2019). Blood-brain barrier: from physiology to disease and back. Physiol. Rev. 99, 21-78. doi: 10.1152/physrev.00050. 2017

Tietz, S., and Engelhardt, B. (2015). Brain barriers: crosstalk between complex tight junctions and adherens junctions. J. Cell Biol. 209, 493-506. doi: $10.1083 /$ jcb. 201412147

Toyama, K., Spin, J. M., and Tsao, P. S. (2017). Role of microRNAs on blood brain barrier dysfunction in vascular cognitive impairment. Curr. Drug Deliv. 14, 744-757. doi: 10.2174/1567201813666160830124627

Villamor, E., Moreno, L., Mohammed, R., Pérez-Vizcaíno, F., and Cogolludo, A. (2019). Reactive oxygen species as mediators of oxygen signaling during fetal-to-neonatal circulatory transition. Free Radic. Biol. Med. 142, 82-96. doi: 10.1016/j.freeradbiomed.2019.04.008

Villas-Bôas, J. M., Maestá, I., and Consonni, M. (2008). Mecanismo de centralização: da insuficiência placentária à adaptação circulatória fetal [Brain sparing effect: from placental insufficiency to fetal circulatory adaptation]. Rev. Bras Ginecol. Obstet. 30, 366-371. doi: 10.1590/S0100-72032008000700008

Wang, Z., Meng, C. J., Shen, X. M., Shu, Z., Ma, C., Zhu, G. Q., et al. (2012). Potential contribution of hypoxia-inducible factor- $1 \alpha$, aquaporin-4, and matrix metalloproteinase-9 to blood-brain barrier disruption and brain edema after experimental subarachnoid hemorrhage. J. Mol. Neurosci. 48, 273-280. doi: 10.1007/s12031-012-9769-6

Warner, D. S., Sheng, H., and Batinić-Haberle, I. (2004). Oxidants, antioxidants and the ischemic brain. J. Exp. Biol. 207, 3221-3231. doi: 10.1242/jeb.01022

Wood, C. E., Rabaglino, M. B., Richards, E., Denslow, N., Zarate, M. A., Chang, E. I., et al. (2014). Transcriptomics of the fetal hypothalamic response to brachiocephalic occlusion and estradiol treatment. Physiol. Genomics. 46, 523-532. doi: 10.1152/physiolgenomics.00186.2013 
Yang, C., Hawkins, K. E., Doré, S., and Candelario-Jalil, E. (2019). Neuroinflammatory mechanisms of blood-brain barrier damage in ischemic stroke. Am. J. Physiol. Cell Physiol. 316, C135-C153. doi: 10.1152/ajpcell.00136.2018

Yang, Y., Estrada, E. Y., Thompson, J. F., Liu, W., and Rosenberg, G. A. (2007). Matrix metalloproteinase- mediated disruption of tight junction proteins in cerebral vessels is reversed by synthetic matrix metalloproteinase inhibitor in focal ischemia in rat. J. Cereb. Blood Flow Metab. 27, 697-709. doi: $10.1038 /$ sj.jcbfm. 9600375

Yang, Y., and Rosenberg, G. A. (2011). Blood-brain barrier breakdown in acute and chronic cerebrovascular disease. Stroke 42, 3323-3328. doi: 10.1161/STROKEAHA.110.608257

Zeng, Y., and Chen, T. (2018). DNA methylation reprogramming during mammalian development. Genes 10:257. doi: 10.3390/genes10040257

Zhao, Z., Nelson, A. R., Betsholtz, C., and Zlokovic, B. V. (2015). Establishment and dysfunction of the bloodbrain barrier. Cell 163, 1064-1078. doi: 10.1016/j.cell.2015.1 0.067
Conflict of Interest: The authors declare that the research was conducted in the absence of any commercial or financial relationships that could be construed as a potential conflict of interest.

Publisher's Note: All claims expressed in this article are solely those of the authors and do not necessarily represent those of their affiliated organizations, or those of the publisher, the editors and the reviewers. Any product that may be evaluated in this article, or claim that may be made by its manufacturer, is not guaranteed or endorsed by the publisher.

Copyright $\odot 2021$ Herrera and González-Candia. This is an open-access article distributed under the terms of the Creative Commons Attribution License (CC BY). The use, distribution or reproduction in other forums is permitted, provided the original author(s) and the copyright owner(s) are credited and that the original publication in this journal is cited, in accordance with accepted academic practice. No use, distribution or reproduction is permitted which does not comply with these terms. 